\title{
Fate of Ising ferromagnets and antiferromagnets by zero-temperature Glauber dynamics on the two-dimensional Archimedean and 2-uniform lattices
}

\author{
Unjong Yu \\ Department of Physics and Photon Science, Gwangju Institute of Science and \\ Technology, Gwangju 61005, Republic of Korea \\ E-mail: uyu@gist.ac.kr
}

\begin{abstract}
The fate of the Ising ferromagnet and antiferromagnet by the zero-temperature Glauber dynamics from random initial spin configuration is investigated in the two-dimensional Archimedean and 2-uniform lattices. Blinker states are found in addition to the ground state and metastable state. We show that an even-coordinated lattice can arrive at a blinker state or a metastable state without stripe structure, in contrast to common expectation. The universal relationship between the critical percolation and the probability of stripe final state is confirmed for six lattices. Results about the fate of the antiferromagnetic Ising model show that the geometric frustration suppresses ordering more and promotes blinker state.
\end{abstract}

Keywords: Kinetic Ising models, Classical Monte Carlo simulations, Coarsening processes. Frustrated systems classical and quantum

\section{Introduction}

The ground state of the ferromagnetic Ising model [1] is simply ferromagnetic phase with all spins in the same direction with one another. It is not trivial, however, when the system is quenched abruptly from a high-temperature disordered phase to below the critical temperature. Especially, quenching to zero-temperature is unique because the system trapped by any finite energy barrier cannot escape from the metastable state forever.

When the system is quenched from infinite temperature (random spin configuration) to zero temperature by the zero-temperature Glauber dynamics, the final spin configuration depends on the spatial dimension: Lower dimensional systems tend to arrive at the ground state more easily. A one-dimensional system always reaches fully ferromagnetic phase in the end [2]. Contrarily, in three-dimensional lattices, complex ferromagnetic domains fluctuate forever, which is called a blinker state 2, 3, 4, 5]. As for complex networks such as the classical random network, the small-world network, and the scale-free network show similar behavior as threedimensional cases [6, 7, 8, 9, 10. It can be understood by their small-world or ultrasmall-world property, which implies an infinite-dimensional system 11. Another notable case is the complete graph, which arrives at the ground state always very 


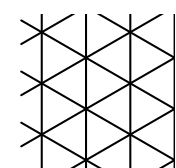

T1

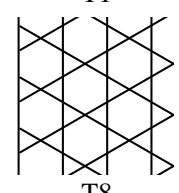

$\mathrm{T} 8$

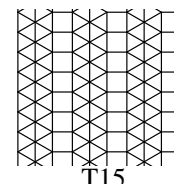

T15

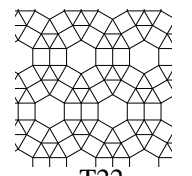

T22

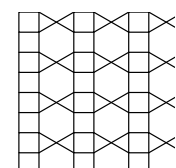

T29

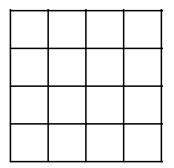

$\mathrm{T} 2$

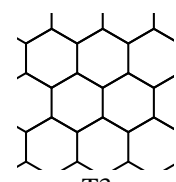

T3

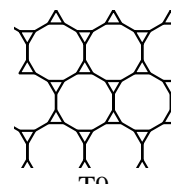

T9

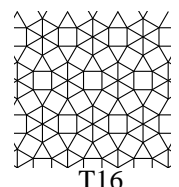

T16

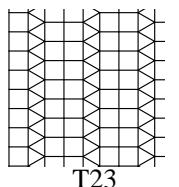

T23

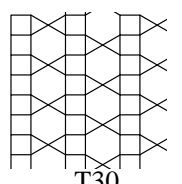

T30

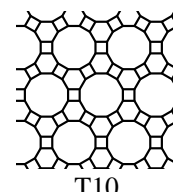

$\mathrm{T} 10$

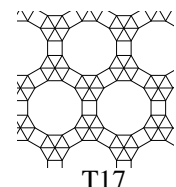

T17

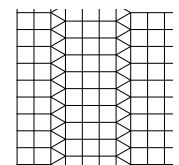

T24

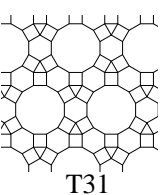

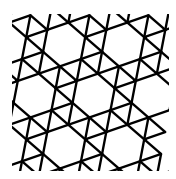

T4

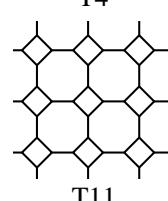

$\mathrm{T} 11$

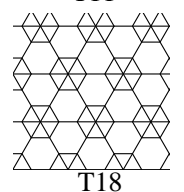

T18

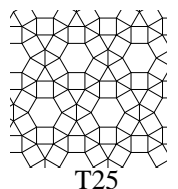

T25

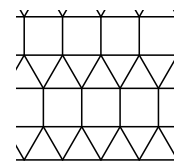

T5

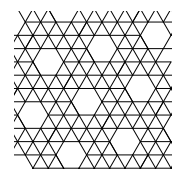

T12
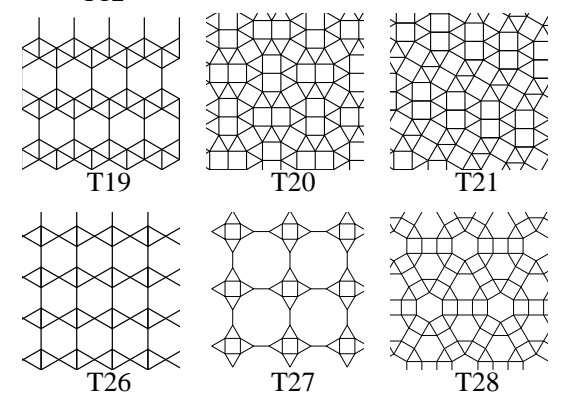

Figure 1. The Archimedean lattices (T1 T11) and the 2-uniform lattices (T12 T 31$)$.

fast [8]. In the complete graph, the shortest-path-length between nodes is always one, and it can be regarded as a zero-dimensional system.

In two-dimensional lattices, which is between one-dimensional and threedimensional cases, the fate depends on the lattice type. For the square and the triangular lattices, the Ising model reaches the ferromagnetic ground state with probability of about $2 / 3$ (for the aspect ratio of one), and the other cases end in metastable states that have straight stripe patterns [2, 3, 4, 12, 13, 14, 15, 16]. As for the honeycomb lattice, it is always trapped in a metastable state 17, 18. It was argued that the fate is determined by whether the coordination number is even or odd [2]. However, the three lattice types are far from enough to make a conclusion considering so many kinds of two-dimensional lattices. In addition, the antiferromagnetic Ising model has not been studied in this context. In the antiferromagnetic model, the geometric frustration, which exists in non-bipartite lattices, changes the transition temperature and even the ground state [19, 20, 21, and it is expected to affect the fate of the zero-temperature Glauber dynamics, too.

In this paper, the fate of the ferromagnetic and antiferromagnetic Ising model under zero-temperature Glauber dynamics from random spin configuration on various two-dimensional lattices is studied. We consider 31 Archimedean and 2-uniform lattices, which are typically used for a systematic study in two dimensions [20, 21, 22, 23. We show that the classification by the coordination number is not always 
Fate of Ising ferromagnets and antiferromagnets

Table 1. Name, coordination number $\left(z_{1}\right.$ and $\left.z_{2}\right)$, and fate of the zerotemperature Glauber dynamics with ferromagnetic $(\mathrm{F})$ and antiferromagnetic (AF) nearest-neighbor interaction in the Archimedean and 2-uniform lattices. For the Archimedean lattices, $z_{2}$ is meaningless because there is only one kind of lattice point. In the two rightmost columns, G, M, and B represent the long-range ordered ground state, metastable state, and blinker state, respectively.

\begin{tabular}{|c|c|c|c|c|c|}
\hline & Name & $z_{1}$ & $z_{2}$ & Fate $(\mathrm{F})$ & Fate(AF) \\
\hline $\mathrm{T} 2$ & $\left(4^{4}\right)$ & 4 & - & $\mathrm{G}+\mathrm{M}$ & - \\
\hline $\mathrm{T} 1$ & $\left(3^{6}\right)$ & 6 & - & $\mathrm{G}+\mathrm{M}$ & B \\
\hline $\mathrm{T} 8$ & $(3,6,3,6)$ & 4 & - & $\mathrm{G}+\mathrm{B}$ & B \\
\hline T30 & $\left(3,4^{2}, 6 ; 3,6,3,6\right)_{2}$ & 4 & 4 & $\mathrm{G}+\mathrm{B}$ & $\mathrm{B}^{\mathrm{a}}$ \\
\hline $\mathrm{T} 26$ & $\left(3^{2}, 6^{2} ; 3,6,3,6\right)$ & 4 & 4 & $\mathrm{G}+\mathrm{M}+\mathrm{B}$ & $\mathrm{G}+\mathrm{M}$ \\
\hline $\mathrm{T} 29$ & $\left(3,4^{2}, 6 ; 3,6,3,6\right)_{1}$ & 4 & 4 & $\mathrm{G}+\mathrm{M}+\mathrm{B}$ & $\mathrm{B}$ \\
\hline$\overline{\mathrm{T} 3}$ & $\left(6^{3}\right)$ & 3 & - & $\mathrm{M}$ & - \\
\hline T10 & $(4,6,12)$ & 3 & - & $\mathrm{M}$ & - \\
\hline $\mathrm{T} 11$ & $\left(4,8^{2}\right)$ & 3 & - & $\mathrm{M}$ & - \\
\hline $\mathrm{T} 4$ & $\left(3^{4}, 6\right)$ & 5 & - & $\mathrm{M}$ & $\mathrm{M}$ \\
\hline T5 & $\left(3^{3}, 4^{2}\right)$ & 5 & - & $\mathrm{M}$ & $\mathrm{M}$ \\
\hline T6 & $\left(3^{2}, 4,3,4\right)$ & 5 & - & $\mathrm{M}$ & $\mathrm{M}$ \\
\hline T9 & $\left(3,12^{2}\right)$ & 3 & - & M & $\mathrm{M}$ \\
\hline $\mathrm{T} 20$ & $\left(3^{3}, 4^{2} ; 3^{2}, 4,3,4\right)_{1}$ & 5 & 5 & $\mathrm{M}$ & $\mathrm{M}$ \\
\hline $\mathrm{T} 21$ & $\left(3^{3}, 4^{2} ; 3^{2}, 4,3,4\right)_{2}$ & 5 & 5 & $\mathrm{M}$ & $\mathrm{M}$ \\
\hline $\mathrm{T} 7$ & $(3,4,6,4)$ & 4 & - & $\mathrm{M}$ & B \\
\hline T13 & $\left(3^{6} ; 3^{4}, 6\right)_{2}$ & 5 & 6 & M & B \\
\hline $\mathrm{T} 17$ & $\left(3^{6} ; 3^{2}, 4,12\right)$ & 4 & 6 & $\mathrm{M}$ & B \\
\hline $\mathrm{T} 18$ & $\left(3^{6} ; 3^{2}, 6^{2}\right)$ & 4 & 6 & $\mathrm{M}$ & B \\
\hline $\mathrm{T} 19$ & $\left(3^{4}, 6 ; 3^{2}, 6^{2}\right)$ & 4 & 5 & M & B \\
\hline $\mathrm{T} 27$ & $\left(3,4,3,12 ; 3,12^{2}\right)$ & 3 & 4 & M & B \\
\hline T31 & $(3,4,6,4 ; 4,6,12)$ & 3 & 4 & $\mathrm{M}$ & B \\
\hline$\overline{\mathrm{T} 12}$ & $\left(3^{6} ; 3^{4}, 6\right)_{1}$ & 5 & 6 & $\mathrm{~B}$ & $\bar{B}$ \\
\hline T14 & $\left(3^{6} ; 3^{3}, 4^{2}\right)_{1}$ & 5 & 6 & $\mathrm{~B}$ & B \\
\hline $\mathrm{T} 15$ & $\left(3^{6} ; 3^{3}, 4^{2}\right)_{2}$ & 5 & 6 & B & B \\
\hline $\mathrm{T} 16$ & $\left(3^{6} ; 3^{2}, 4,3,4\right)$ & 5 & 6 & B & B \\
\hline $\mathrm{T} 22$ & $\left(3^{3}, 4^{2} ; 3,4,6,4\right)$ & 4 & 5 & $\mathrm{~B}$ & B \\
\hline $\mathrm{T} 23$ & $\left(3^{3}, 4^{2} ; 4^{4}\right) 1$ & 4 & 5 & B & B \\
\hline $\mathrm{T} 24$ & $\left(3^{3}, 4^{2} ; 4^{4}\right)_{2}$ & 4 & 5 & $\mathrm{~B}$ & B \\
\hline $\mathrm{T} 25$ & $\left(3^{2}, 4,3,4 ; 3,4,6,4\right)$ & 4 & 5 & $\mathrm{~B}$ & B \\
\hline $\mathrm{T} 28$ & $\left(3,4^{2}, 6 ; 3,4,6,4\right)$ & 4 & 4 & $\mathrm{~B}$ & B \\
\hline
\end{tabular}

${ }^{a}$ Blinker state with partial one-directional long-range ordering.

correct, and a blinker state can be found also in two-dimension.

\section{Model and methods}

The energy of the Ising model is defined by the following Hamiltonian.

$$
H=-J \sum_{\langle i, j\rangle} S_{i} S_{j}
$$

The spin at the $i$-th site $S_{i}$ may take the values of +1 (up) or -1 (down), only. The summation $\langle i, j\rangle$ runs for all the nearest-neighbor spin pairs. Positive and negative $J$ represent ferromagnetic and antiferromagnetic interactions, respectively. The simulation starts from a random spin configuration, which corresponds to the infinite temperature. The beginning temperature is not important if it is higher than the transition temperature. The case where the system starts from the transition 
temperature is studied in [16. In the zero-temperature Glauber dynamics, a spin is randomly chosen and the spin is flipped if the flipping reduce the energy. When there is no change in energy by the spin flipping, it is flipped with probability $1 / 2$. If a spin has the same direction as the majority of its neighbors, the spin does not change its direction (type-I); in the opposite case, the spin changes the direction when chosen (type-II); if the two directions tie, the spin may be flipped or not (typeIII). Interestingly, the zero-temperature Glauber dynamics is exactly the same as the majority-vote model without noise [24]. The difference between the two models is the interpretation of the metastable state. When all the spins are of type-I without fully ferromagnetic order, it is a metastable state with higher energy than the ground state in the Ising model, but all the spins (agents) are satisfied without frustration in the majority-vote model.

To accelerate the simulation, the continuous-time Monte-Carlo method [25] was used. Two lists are maintained for spins of type-II and type-III, respectively. When the size of the two lists are $N_{\text {II }}$ and $N_{\text {III }}$, one spin is chosen randomly with probability $2 /\left(2 N_{\text {II }}+N_{\text {III }}\right)$ and $1 /\left(2 N_{\text {II }}+N_{\text {III }}\right)$ in the lists of type-II and type-III, respectively. The chosen spin is flipped and the lists are updated. By this method, fruitless selection of type-I spin is avoided. The simulation stops when there is no spin in the lists (ground state or metastable state) or all the spins of type-III are blinker spins, which flips forever, with $N_{\text {II }}=0$ (blinker state). The simulation is repeated $2 \times 10^{4}$ times for each case and the final states are classified into the ground state, metastable state, and blinker state.

In this work, lattices made by edge-to-edge tiling by regular polygons are considered. When a lattice has $k$ kinds of lattice points with respect to the polygons surrounding the point, it is called a $k$-uniform lattice. There are only eleven 1-uniform lattices, also called the Archimedean lattices, and twenty 2-uniform lattices [26, which are listed in Table 1 and Fig. 1. The periodic boundary condition is used. The shape of clusters is rectangle with aspect ratio $r=l_{y} / l_{x}$, where $l_{x}$ and $l_{y}$ are the lengths of the cluster in the $x$ and $y$ directions, respectively.

\section{Results and discussion}

The fate of the 31 lattices considered here can be classified into three groups. Lattices of the first group arrive at the ground state or a stripe phase in the end. Six lattices including the triangular (T1) and the square (T2) lattices belong to this group. The stripe phase is a metastable state for the triangular and the square lattices, as is known already [2]. In T8 and T30, blinker spins separate the two domains in a stripe state, as shown in Fig. 2 Isolated blinker spins, which are surrounded by stationary spins unaffected by the sign of the blinker spins, cannot move or expand, and fluctuate forever. They can appear alone like in Fig. 2(b)-(e) or in pairs like in diagonal stripe state of T30 [Fig. 2(f)]. Single blinker spins fluctuate between up and down spins, and blinker pairs in T30 wander among $(\uparrow \uparrow),(\uparrow \downarrow)$, and $(\downarrow \downarrow)$ states. In the case of T26 and T29, the final state can be a blinker state or a metastable state depending on the direction of the stripes.

Among the six lattices of the first group, three lattices (T2, T26, and T29) may have stripe phase in the two directions (horizontal and vertical directions) and the probabilities of them $\left(P_{=}\right.$and $\left.P_{\|}\right)$depend on the aspect ratio $r$. [See Fig. [3(a).] As for the square lattice (T2), the dependence on the aspect ratio was proposed to have relation to the critical percolation [14, which is confirmed again in this work. 


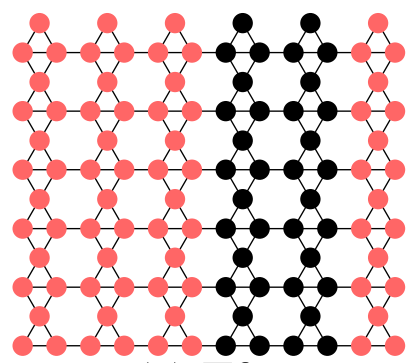

(a) $\mathrm{T} 26$

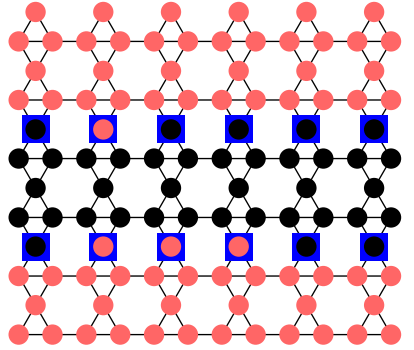

(b) $\mathrm{T} 26$

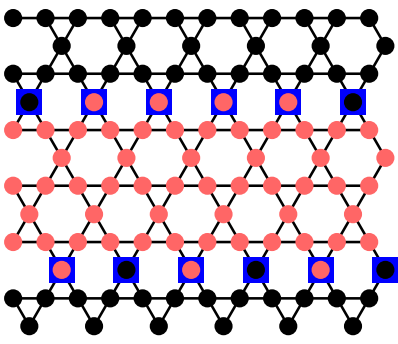

(c) $\mathrm{T} 8$

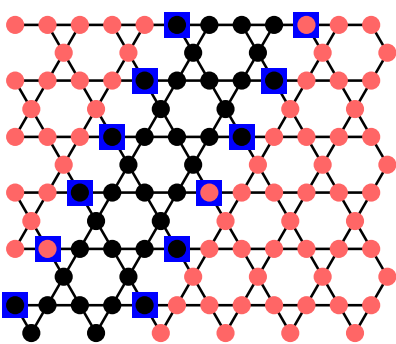

(d) $\mathrm{T} 8$

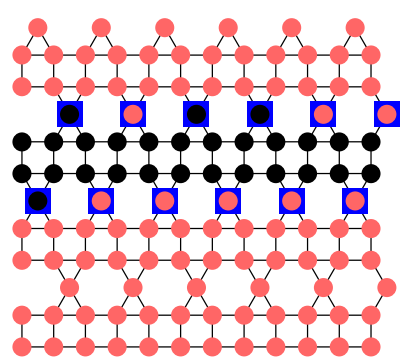

(e) $\mathrm{T} 30$

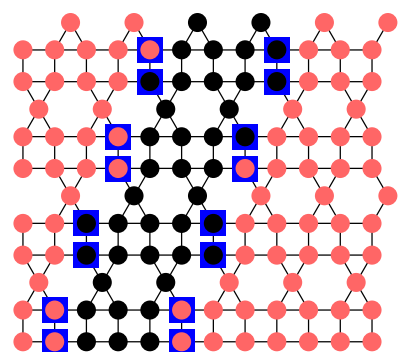

(f) $\mathrm{T} 30$

Figure 2. Typical final spin configurations with stripes in the ferromagnetic Ising model. Up-spins, down-spins, and blinker spins are expressed by black circles, red circles, and blue squares, respectively.
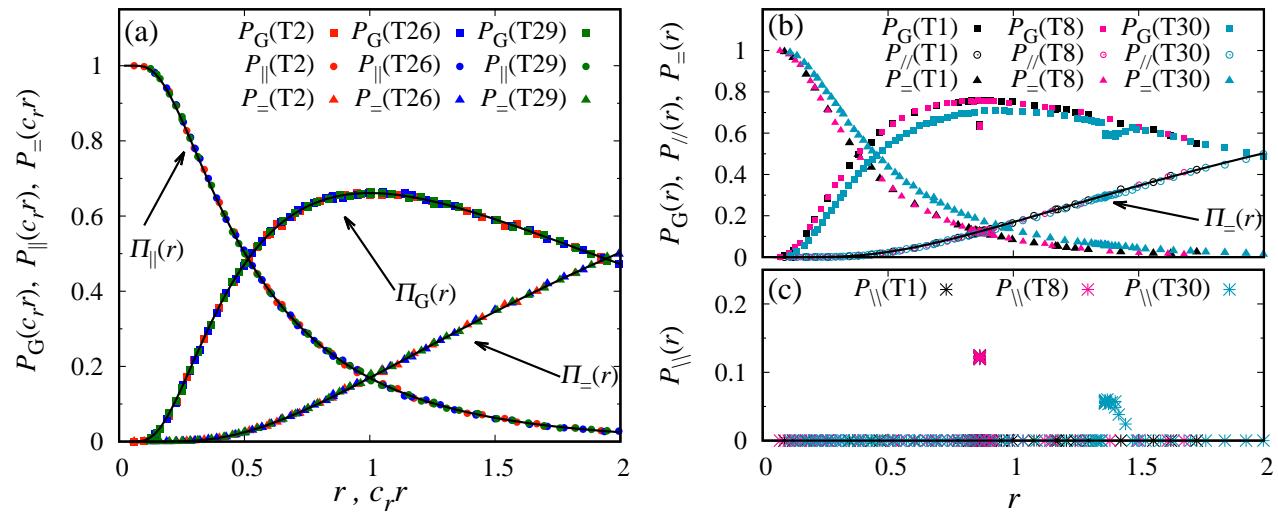

Figure 3. The probabilities for final state of the zero-temperature Glauber dynamics as a function of the aspect ratio $r=l_{y} / l_{x} . P_{\mathrm{G}}, P_{\|}, P_{=}, P_{/ /}$, and $P_{\backslash \backslash}$ mean the probabilities of the ground state, and stripe states in the vertical, horizontal, and diagonal directions. Rescaling factor $c_{r}$ was obtained by fitting to be 0.822(2) and 0.920(1) for T26 and T29, respectively. For the other lattices, $c_{r}=1$ within statistical error. Black solid lines represent the winding probability of the critical percolation in the vertical and horizontal directions $\left[\Pi_{\|}(r)\right.$ and $\left.\Pi_{=}(r)\right]$ and the other cases $\left[\Pi_{\mathrm{G}}(r)=1-\Pi_{\|}(r)-\Pi_{=}(r)\right]$. The number of spins $(N)$ is between 600 and 480000 . The error bars are of similar size as the data points.

Soon after a zero-temperature quenching, the system approaches the critical phase quickly [27, 28, and a percolated cluster evolves to a stripe domain when it exists. 


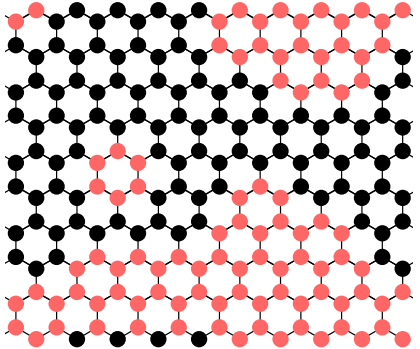

(a) T3

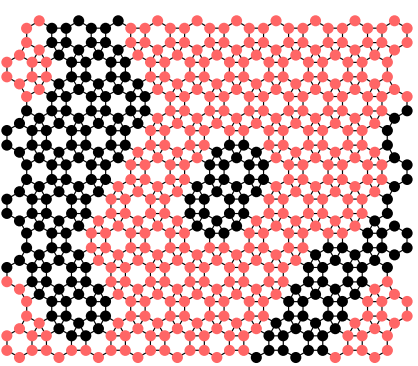

(b) $\mathrm{T} 7$

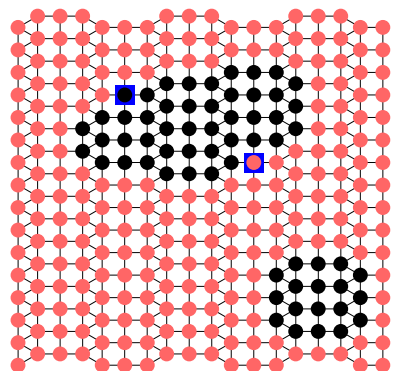

(c) $\mathrm{T} 16$

Figure 4. Typical final spin configurations of a metastable state in (a) and (b), and of a blinker state in (c). Up-spins, down-spins, and blinker spins are expressed by black circles, red circles, and blue squares, respectively.

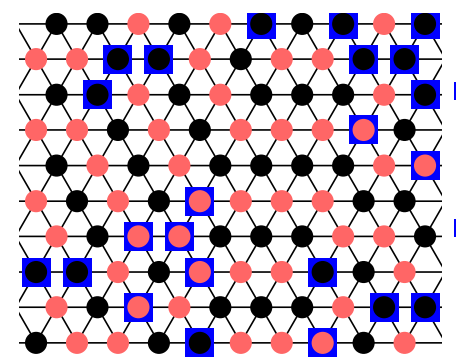

(a) $\mathrm{T} 1$

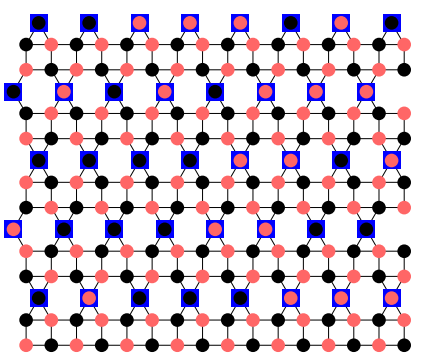

(b) T30

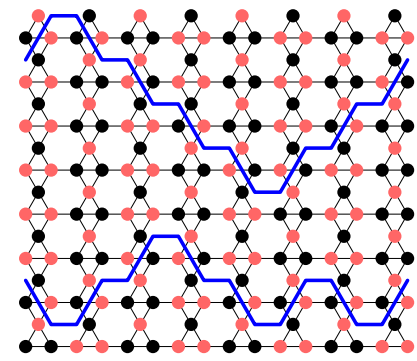

(c) $\mathrm{T} 26$

Figure 5. Typical final spin configurations in the antiferromagnetic Ising model for T1, T30, and T26. Up-spins, down-spins, and blinker spins are expressed by black circles, red circles, and blue squares, respectively. In (c), domain boundary is represented by blue thick lines.

Therefore, the probability of the final stripe phase in a certain direction is the same as the probability of percolation in the direction at the critical percolation, which is approximated as follows [29, 14].

$$
\begin{aligned}
& \Pi_{\|}(r)=1-2 \rho^{5 / 4}+\rho^{3}+2 \rho^{12}-4 \rho^{5 / 43}+3 \rho^{15} \\
& \Pi_{=}(r)=\sqrt{\frac{2 r}{3}}\left(\rho^{3}-\rho^{15}-\rho^{27}+4 \rho^{35}\right) \\
& \text { with } \rho=e^{-\pi / 6 r}
\end{aligned}
$$

where $\Pi_{\| \mid}(r)\left(\Pi_{=}(r)\right)$ is the universal probability of single or multiple separate windings in the vertical (horizontal) direction, but not in the other direction for a rectangle of aspect ratio $r$. The other kinds of domains are unstable and so the probability to arrive at the ground state is $\Pi_{\mathrm{G}}(r)=1-\Pi_{||}(r)-\Pi_{=}(r)$. Equations (2)-(44) are accurate at least within $0.1 \%[29]$ and are good enough to compare with our results. Figure 3(a) shows that the agreement between the simulation results and the analytic results is excellent. For T26 and T29, which have different property in the horizontal and the vertical directions, a rescaling factor $c_{r}$ is needed to apply the analytic results derived for the square lattice. They were obtained by fitting of $\Pi_{\eta}(r)=P_{\eta}\left(c_{r} r\right)$ to be $c_{r}=0.822(2)$ and $0.920(1)$ for T26 and T29, respectively. The other three lattices (T1, T8, and T30) may have stripe states in three directions in addition to 
the ground state. By the restriction of the periodic boundary condition, however, stripes of only two directions are possible in general. As shown in Fig. 3(c), in the boundary condition used here, stripe states of the third direction are allowed only at $r=\sqrt{3} / 2$ for $\mathrm{T} 1$ and $\mathrm{T} 8$, and near $r=(1+\sqrt{3}) / 2$ for T30. For other values of aspect ratio, the probability of stripe states in that direction becomes zero; this is reflected in many data of $P_{\backslash \backslash}(r)=0$ in Fig. 3(c). For lattices with diagonal stripe states, only the horizontal stripe phase can be compared with the analytic solution of the critical percolation [16]. As shown in Fig. 3(a)-(c), the dependence on the cluster size is not observed for $N \gtrsim 600$, where $N$ is the number of spins within the cluster.

Lattices of the second and the third groups result in metastable state and blinker state, respectively. The honeycomb lattice (T3) has a metastable final state, as is known [17, 18. Typical metastable and blinker states as a fate are shown in Fig. 4. The boundary of domains can bend and an isolated domain island can exist inside of a domain of the opposite spin. Therefore, the possibility of the ground state becomes zero as the cluster size increases. Depending on the details of the lattice structure, a blinker spin can appear at the interface. The probability of blinker spins also increases with the cluster size if it is possible to appear. Stripe states can also appear with metastable or blinker states. 18

The classification of fate is summarized in Table 1. When a lattice includes oddcoordinated lattice points, it always belongs to the second or the third group, and cannot arrive to the ground state. Only even-coordinated lattices can belong to the first group. However, even-coordinated lattices are not always in the first group: we found a few even-coordinated lattices (T7, T17, T18, and T28) that always fall into a metastable state or a blinker state, against common expectation.

We also investigated the fate of the antiferromagnetic Ising model on the 31 lattices to study the frustration effect on the zero-temperature Glauber dynamics. Bipartite lattices (T2, T3, T10, and T11), which do not have frustration, behave exactly the same way as the ferromagnetic case; in non-bipartite lattices, however, frustration tends to suppress the order [19. While weak frustration just reduces the transition temperature, strong frustration eliminates long-range order even at zero temperature resulting in the spin ice or the spin liquid phase [20, 21]. Both of spin ice and spin liquid do not have long-range order, but the former is frozen below freezing temperature while the latter fluctuates forever even at zero temperature. Therefore, the spin liquid phase is similar to the blinker state. The triangular (T1) and the kagome (T8) lattices, which are spin liquid at the ground state even by slow cooling [20], become blinker state as is expected. [See Fig. 5(a).] A lattice that has a blinker final state in the ferromagnetic Ising model, always becomes a blinker state in the antiferromagnetic case; A lattice of a metastable final state in the ferromagnetic case may become a metastable state or a blinker state with antiferromagnetic interaction. The results are summarized in Table 1 .

Most of non-bipartite lattices have a metastable or a blinker state without longrange ordering, but there are two notable exceptions: T30 and T26. As shown in Fig. [5(b), T30 always has a stripe pattern in the final state: long-range-ordered twoleg ladders are separated by a string of blinker spins. Since interactions between the ladders are blocked by blinker spins, each ladder can be considered as one-dimensional effectively, and full long-range ordering is realized on the ladders. T26 is the only non-bipartite lattice that may have the complete long-range-ordered ground state as a fate in the antiferromagnetic Ising model among lattices considered here. It may also have three kinds of stripe state in horizontal and two diagonal directions. 
Vertical stripe states are unstable and always result in the ground state. As shown in Fig. 5 (c), a horizontal stripe is not straight but in zigzags. As for the aspect ratio of $\sqrt{3} / 2$, where all the three stripe structures are possible, $P_{G}=0.900(2)$, $P_{=}=0.069(1), P_{/ /}=0.016(1), P_{\|}=0.015(1)$. Size dependence was not noticed for $2700 \leq N \leq 874800$.

\section{Summary}

The fate of the Ising model by quenching through the zero-temperature Glauber dynamics is investigated in the 31 two-dimensional lattices (Archimedean and 2uniform lattices). They are classified into three groups: (i) the ground state and stripe states, (ii) a metastable state, and (iii) a blinker state. As for the ferromagnetic Ising model, contrary to common expectation that even-coordinated and odd-coordinated lattices belong to the group (i) and (ii), respectively, a blinker state was found to exist in two dimensions. In addition, we showed that even-coordinated lattice can belong to the group (ii) or (iii) to have a metastable or a blinker state without stripe structure as final state. The universal relationship between the critical percolation and the probability of striped final state was confirmed for six lattices. Study of the antiferromagnetic Ising model showed that frustration suppresses ordering more and promotes blinker state rather than metastable and long-range ordered state.

\section{Acknowledgments}

This work was supported by GIST Research Institute (GRI) grant funded by the GIST in 2017.

\section{References}

[1] E. Ising. Beitrag zur Theorie des Ferromagnetismus. Z. Phys., 31(1):253-258, 1925.

[2] V. Spirin, P. L. Krapivsky, and S. Redner. Fate of zero-temperature Ising ferromagnets. Phys. Rev. E, 63(3):036118, 2001.

[3] V. Spirin, P. L. Krapivsky, and S. Redner. Freezing in Ising ferromagnets. Phys. Rev. E, 65(1):016119, 2001.

[4] P. Sundaramurthy and D. L. Stein. Zero-temperature dynamics of 2D and 3D Ising ferromagnets. J. Phys. A: Math. Gen., 38(2):349-362, 2005.

[5] J. Olejarz, P. L. Krapivsky, and S. Redner. Zero-temperature relaxation of three-dimensional Ising ferromagnets. Phys. Rev. E, 83(5):051104, 2011.

[6] O. Häggström. Zero-temperature dynamics for the ferromagnetic Ising model on random graphs. Physica A, 310(3-4):275-284, 2002.

[7] D. Boyer and O. Miramontes. Interface motion and pinning in small-world networks. Phys. Rev. E, 67(3):035102, Mar 2003.

[8] C. Castellano, V. Loreto, A. Barrat, F. Cecconi, and D. Parisi. Comparison of voter and Glauber ordering dynamics on networks. Phys. Rev. E, 71(6):066107, Jun 2005.

[9] C. Castellano and R. Pastor-Satorras. Zero temperature Glauber dynamics on complex networks. J. Stat. Mech.: Theory Exp., 2006(05):P05001, 2006.

[10] C. P. Herrero. Zero-temperature Glauber dynamics on small-world networks. J. Phys. A: Math. Theor., 42(41):415102, 2009.

[11] L. Daqing, K. Kosmidis, A. Bunde, and S. Havlin. Dimension of spatially embedded networks. Nat. Phys., 7(6):481-484, 2011.

[12] A. Lipowski. Anomalous phase-ordering kinetics in the Ising model. Physica A, 268(1):6-13, 1999.

[13] P. M. C. de Oliveira, C. M. Newman, V. Sidoravicious, and D. L. Stein. Ising ferromagnet: zero-temperature dynamic evolution. J. Phys. A: Math. Gen., 39(22):6841, 2006. 
[14] K. Barros, P. L. Krapivsky, and S. Redner. Freezing into stripe states in two-dimensional ferromagnets and crossing probabilities in critical percolation. Phys. Rev. E, 80(4):040101, 2009.

[15] J. Olejarz, P. L. Krapivsky, and S. Redner. Fate of 2D kinetic ferromagnets and critical percolation crossing probabilities. Phys. Rev. Lett., 109(19):195702, Nov 2012.

[16] T. Blanchard and M. Picco. Frozen into stripes: Fate of the critical Ising model after a quench. Phys. Rev. E, 88(3):032131, Sep 2013.

[17] H. Takano and S. Miyashita. Ordering process in the kinetic Ising model on the honeycomb lattice. Phys. Rev. B, 48(10):7221-7226, Sep 1993.

[18] T. Blanchard, L. F. Cugliandolo, M. Picco, and A. Tartaglia. Critical percolation in the dynamics of the $2 \mathrm{~d}$ ferromagnetic Ising model. arXiv:1705.06508v3.

[19] R. Moessner and A. P. Ramirez. Geometrical frustration. Phys. Today, 59(2):24, 2006.

[20] U. Yu. Ising antiferromagnet on the Archimedean lattices. Phys. Rev. E, 91(6):062121, Jun 2015.

[21] U. Yu. Ising antiferromagnet on the 2-uniform lattices. Phys. Rev. E, 94(2):022112, Aug 2016.

[22] J. Richter, J. Schulenburg, and A. Honecker. Quantum magnetism in two dimensions: From semi-classical Néel order to magnetic disorder. In U. Schollwöck, J. Richter, D. J. J. Farnell, and R. F. Bishop, editors, Quantum Magnetism, volume 645 of Lecture Notes in Physics. Springer, Berlin, 2004.

[23] R. A. Neher, K. Mecke, and H. Wagner. Topological estimation of percolation thresholds. J. Stat. Mech.: Theory Exp., 2008(01):P01011, 2008.

[24] M. J. de Oliveira. Isotropic majority-vote model on a square lattice. J. Stat. Phys., 66(1):273$281,1992$.

[25] A. B. Bortz, M. H. Kalos, and J. L. Lebowitz. A new algorithm for Monte Carlo simulation of Ising spin systems. J. Comput. Phys., 17(1):10-18, 1975.

[26] B. Grünbaum and G. C. Shephard. Tilings and Patterns. W. H. Freeman and Company, New York, 1987.

[27] J. J. Arenzon, A. J. Bray, L. F. Cugliandolo, and A. Sicilia. Exact results for curvature-driven coarsening in two dimensions. Phys. Rev. Lett., 98(14):145701, Apr 2007.

[28] A. Sicilia, J. J. Arenzon, A. J. Bray, and L. F. Cugliandolo. Domain growth morphology in curvature-driven two-dimensional coarsening. Phys. Rev. E, 76(6):061116, Dec 2007.

[29] G. Pruessner and N. R. Moloney. Winding clusters in percolation on the torus and the Möbius strip. J. Stat. Phys., 115(3):839-853, 2004. 